\title{
Cyclic Biaxial Stress Measurement Using EBSD Analysis of Grown Grains Occurred in Copper Foil
}

\author{
Yuichi $\mathrm{ONO}^{1 *}$ \\ ${ }^{1}$ Department of Mechanical and Aerospace Engineering, Tottori University, 4-101 Koyama-cho, Minami, Tottori-shi, Tottori, Japan \\ *ono@mech.tottori-u.ac.jp
}

\begin{abstract}
Keywords: Stress-Strain Measurement, Fatigue, Electron Back Scatter Diffraction, Combined Stress, Copper Electroplating Method
\end{abstract}

Introduction: A cyclic biaxial stress measurement method using electrodeposited copper foil ${ }^{(1)(2)}$ was examined. The crystallographic orientations of individual grains that undergo grain growth in copper foil subjected to cyclic loading were analyzed by electron backscatter diffraction $(\mathrm{EBSD})^{(3)}$. Then, the biaxial stress dependence of grain orientation was examined using the orientation imaging microscopy (OIM) mapping. And, a biaxial stress measurement method using the pole figure and the OIM mapping were proposed for negative biaxial stress ratios.

Experimental Procedures: The copper foil was adhered to a strip of titanium alloy, and cyclic loading tests were carried out using the Schenck type fatigue testing machine under various biaxial stress conditions. The crystallographic orientations of grown grains were analyzed using scanning electron microscopy with electron backscatter diffraction (SEM-EBSD) equipment (Hitachi S-3100H).

Results and Discussion: Most grown grains were oriented with the $\{110\}$ plane parallel to the foil surface, and one of the grown grain slip direction corresponded closely to the first principal shearing stress (maximum shearing) direction. These experimental results suggest that the angle $\psi$ between the longitudinal direction and the straight line connecting two circumferential high-density areas in the $\{110\}$ pole figure corresponded to the first principal shearing stress direction (Fig. 1). Therefore, the biaxial stress ratio $C$ can be obtained by measuring this angle (Fig.2). Moreover, the number of grains with slip directions that did not correspond to the direction of first principal shearing stress increased as the biaxial stress ratio approached zero. We consider that the main reason for this was the second principal shearing stress acting in the thickness direction. According this feature, anther stress measurement method was introduced by using OIM mapping. The ratio $r_{r+b}$ of grain area with slip directions not corresponding to the first principal shearing stress direction to the total grain growth area in OIM mapping was measured by the image processing software. This ratio increased when biaxial stress ratio exceeded -0.32 (Fig. 3). Therefore, if $C$ is in the range from -0.32 to 0 , biaxial stress measurement is possible. These new methods can measure the principal stresses in an area of $500 \mu \mathrm{m} \times 500 \mu \mathrm{m}$. This area corresponds to the resolution of this method.

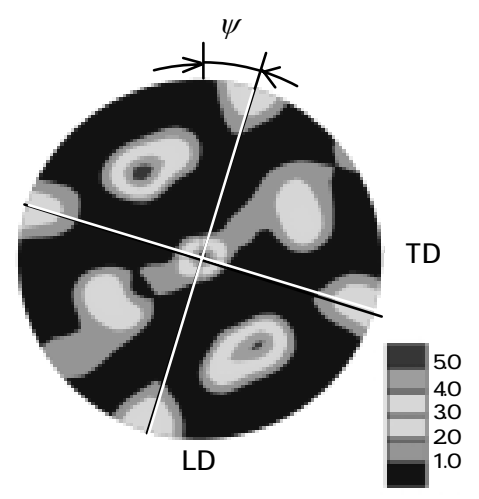

Fig. $1\{110\}$ pole figure

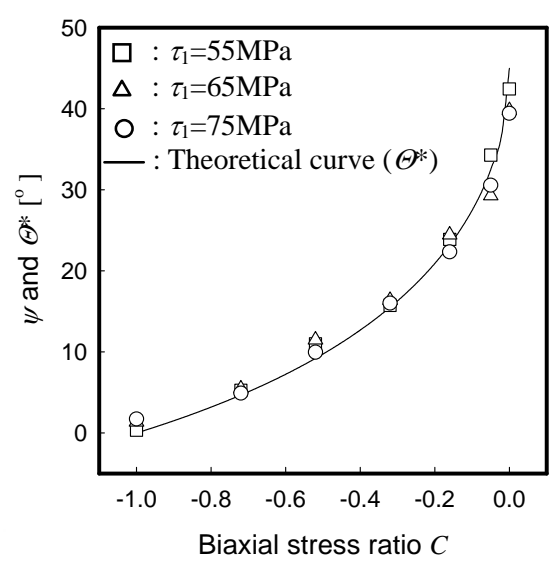

Fig.2 Relationship between $\psi$ and $C$

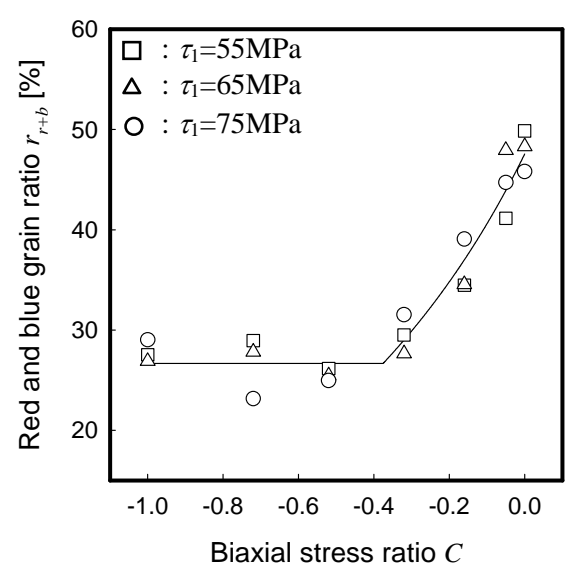

Fig.3 Relationship between $r_{r+b}$ and $C$

\section{References}

(1) Kato, A., Stress Measurement by Copper Electroplating Based on Grain Growth, Experimental Mechanics, Vol.35, No.1(1995), pp.24-30.

(2) Ono, Y. and Kitaoka, S., Cyclic Stress Measurement Method Using Grain Size and Occurrence Rate of Grown Grains in Electrodeposited Copper Foil, Strain, Vol.47, No.2(2011), pp.154-161.

(3) Engler, O. and Randle, V., Introduction to Texture Analysis, 2nd edition, (2010), pp.15-50, CRC Press. 